Ann. Biol. anim. Bioch. Biophys., I975, 15 (4), 757-763.

\title{
ÉTUDES PROSPECTIVES DES FACTEURS CHROMOSOMIQUES DE QUALITÉ DES GAMETES
}

\author{
Joëlle BOUÉ et A. BOUÉ \\ C. E. B. I. O. P., I. N. S. E. R. M., U. 73, Château de Longchamp,
} 75016 Paris

\section{RÉSUMÉ}

Il est possible d'étudier les facteurs chromosomiques agissant sur la qualité des gamètes chez les porteurs d'une anomalie chromosomique structurale balancée. La consultation de génétique pour le diagnostic prénatal permet de suivre l'évolution des grossesses de ces couples.

L'analyse des premiers résultats indique :

I. Le développement de la plupart des zygotes déséquilibrés s'arrête rapidement et ils sont éliminés avant la $\mathrm{r} 6^{\mathrm{e}}$ semaine de gestation.

2. $\mathrm{I} / \mathrm{r}^{\mathrm{e}}$ seulement des porteurs de translocations réciproques et robertsoniennes sont des mâles, ce qui semble indiquer que ces anomalies ont des conséquences plus graves chez le mâle : oilgospermie, stérilité, avortements précoces. Ceci est illustré par les observations faites dans les cas de translocation I $4 q 2$ I $q$ et dans les inversions péricentriques des chromosomes 2 et 9 .

Les travaux de ces dernières années ont apporté une vue d'ensemble des échecs de la reproduction humaine d'origine chromosomique. La responsabilité des anomalies chromosomiques a été montrée dans des malformations congénitales compatibles avec la vie ou conduisant à une mortalité périnatale, et surtout dans les avortements spontanés précoces; l'analyse de ces résulitats a permis d'évaluer la grande fréquence des conceptions avec des anomalies entraînant des arrêts très précoces du développement, éliminées avant que la grossesse soit reconnue, leur répétition pouvant alors simuler une stérilité $[\mathrm{I}, 2,3]$.

Ce sont les anomalies numériques résultant d'accidents survenant au cours de la gamétogenèse ou au moment de la fécondation qui sont le plus souvent en cause.

Les études biologiques et épidémiologiques faites dans les avortements spontanés ont déjà permis de préciser certains facteurs favorisant les accidents numériques et les conséquences de ces anomalies sur la croissance cellulaire et le dévelop- 
pement $[4,5]$. Le caractère accidentel de ces anomalies du nombre des chromosomes, l'impossibilité de préciser régulièrement si les accidents de non-disjonction sont produits dans la gamétogenèse mâle ou femelle, limitent la réalisation d'études prospectives rigoureuses.

Les parents porteurs d'anomalies de la structure chromosomique constituent un groupe avec un risque particulièrement élevé d'échecs de la reproduction. Depuis l'introduction des techniques d'identification chromosomique par bandes, il est possible d'aborder avec une grande précision l'étude de ces anomalies. En outre, l'analyse chromosomique des cellules foetales prélevées par ponction amniotique précoce permet à ces couples d'envisager de nouvelles grossesses. Il est donc possible dans un seul laboratoire et dans un temps raisonnable de recueillir un nombre suffisant d'observations pour réaliser une étude prospective.

Une cinquantaine de grossesses ont été suivies chez des couples où l'un des géniteurs est porteur d'une anomalie de la structure chromosomique. Une consultation de génétique a lieu soit avant la conception, soit en tout début de grossesse, ceci permet de suivre les premières semaines de la grossesse sur le plan clinique et biologique, toute thérapeutique hormonale étant exclue. Les éventuels avortements spontanés sont étudiés et à la $I 7^{\mathrm{c}}$ semaine gestationnelle est pratiqué une ponction amniotique permettant l'analyse des cellules fotales. Ce programme est évidemment difficile à appliquer dans tous les cas, certains couples n'étant adressés au laboratoire que peu de temps avant la ponction.

Dans cet exposé sont présentés les résultats cliniques et chromosomiques, l'analyse des dosages hormonaux urinaires et plasmatiques ne pourra être faite que lorsque le nombre des observations sera plus important.

Les techniques de cultures des cellules amniotiques et des tissus d'avortements, les techniques chromosomiques ont déjà été publiées $[6,7]$.

\section{RÉSULTATS}

Le tableau I donne les résultats succincts de 1'analyse chromosomique de 38 diagnostics intra-utérins. On remarque immédiatement qu'alors que le risque théorique de conceptions avec anomalies est particulièrement élevé dans cette population, il n'a été décelé que deux anomalies déséquilibrées : une trisomie $2 \mathbf{I}$ par translocation I4 $\sim 2 \mathrm{I}$; une aneušomie de recombinaison consécutive à une inversion péricentrique du chromosome 3 [8].

Pour expliquer ces résultats on est amené à penser que les anomalies déséquilibrées doivent conduire le plus souvent à un arrêt précoce du développement du zygote et sont éliminées avant la date de la ponction amniotique. Ceci correspond aux observations faites dans les avortements spontanés où la plupart des anomalies chromosomiques conduisent à un arrêt du développement avant la $7^{\text {e semaine après }}$ la conception et une expulsion qui se situe le plus souvent avant la $\mathbf{I}^{\mathbf{e}}$ semaine gestationnelle $[2,6]$.

Il a été possible de confirmer cette hypothèse dans un groupe homogène d'anomalie de la structure, la translocation par fusion centrique $I_{4} \sim 2 \mathrm{I}$. Vingt grossesses 
chez des couples où l'un des parents était porteur de cette anomalie ont été suivies : 7 avortements spontanés ont eu lieu avant la date de la ponction, 4 ont pu être recueillis et étudiés et chaque fois une trisomie 2 I a été diagnostiquée ; I3 grossesses ont évolué normalement jusqu'à la $\mathbf{I}^{\mathbf{e}}$ semaine et le caryotype du fœtus a été établi sur les cellules prélevées par ponction amniotique.

\section{TABLEAU I}

Résultats de l'analyse chromosomique des cellules foetales prélevées par ponction amniotique lorsqu'un des parents est porteur d'une anomalie de la structuve chromosomique

\begin{tabular}{|c|c|c|c|}
\hline \multirow{4}{*}{ Caryotype du géniteur } & \multicolumn{3}{|c|}{ Caryotype fœtal } \\
\hline & \multirow{2}{*}{\multicolumn{2}{|c|}{ Phénotype normal }} & \multirow{3}{*}{ Anomalies } \\
\hline & & & \\
\hline & Normal & Equuilibré & \\
\hline \multicolumn{4}{|l|}{ Translocation Robertsonienne } \\
\hline $\mathrm{Iq}_{\mathrm{q}} \mathrm{Dq}$ & 2 & 5 & \\
\hline $\mathrm{Dq} \mathrm{Gq}$ & 6 & 6 & 1 Trisomie 21 \\
\hline Translocation Réciproque & 5 & 6 & \\
\hline Inversion Péricentrique & 3 & 4 & 1 Aneusomie (3) \\
\hline Insertion & 1 & & \\
\hline
\end{tabular}

Le tableau 2 donne les résultats de ces diagnostics : douze enfants sont normaux 6 portent la translocation équilibrée et 6 ont un caryotype normal; une trisomie $2 \mathrm{I}$ par translocation a été décelée.

TABLEAU 2

Evolution de la grossesse et caryotype des cellules foetales lorsqu'un des parents est porteur d'une translocation $14 \sim 21$

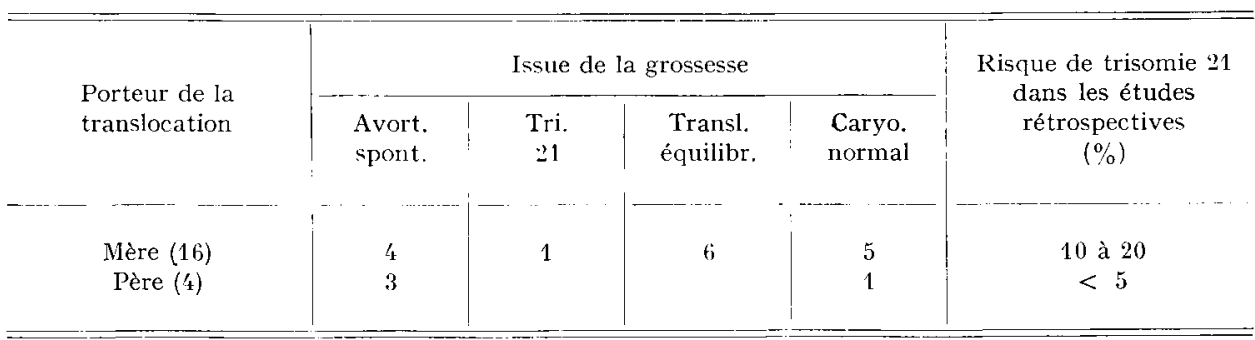

Bien que limitée cette série permet d'apporter des explications aux divergences apparentes entre les probabilités théoriques et les observations faites à la naissance. On doit d'abord noter que les monosomies I4 et 2 I n'ont jamais été signalées dans 
des avortements précoces, elles doivent être éliminées très tôt sans que la mère ait la notion de la conception, la trisomie I4 est toujours létale et conduit à des avortements précoces, il est possible que cette anomalie ait été à l'origine de certains des avortements non caryotypés. Sur les I 7 grossesses pour lesquelles un examen chromosomique a été possible, nous avons 5 trisom1es 2 I ; cette évaluation des trisomies 2 I qui ne peut être qu'un chiffre minimum est en accord avec la probabilité théorique, une seule trisomie $2 \mathrm{I}$ a évolué au-delà de la $\mathrm{I} 7^{\mathrm{e}}$ semaine. I es avortements spontanés de trisomies 2 I expliquent donc les divergences apparentes entre la fréquence observée à la naissance et le risque théorique.

Une différence très importante apparaît lorsque les résultats sont analysés en fonction du géniteur porteur de l'anomalie.

Dans les 3I diagnostics faits pour une translocation (par fusion centrique ou réciproque), trois fois seulement 1'anomalie était portée par le père (tabl. 3).

\section{TABLEAU 3}

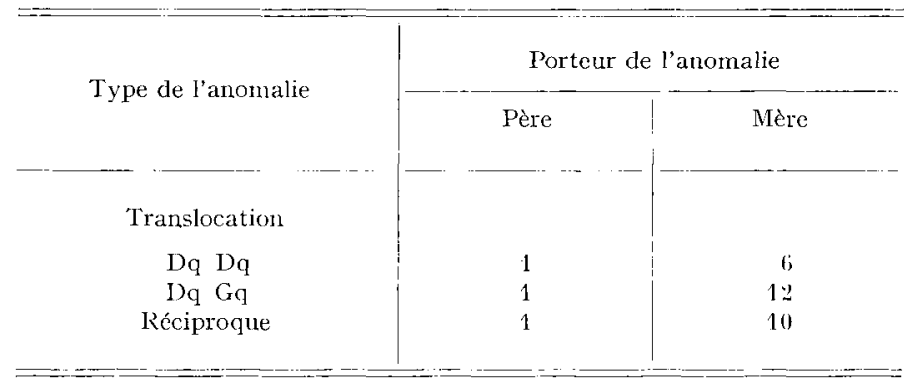

L'analyse des grossesses chez les couples où l'un des géniteurs porte une translocation I $4 \sim 2$ I équilibrée apporte des précisions (tabl. 2). Lorsque la mère porte la translocation il a été observé I trisomie 2 I évoluant au-delà de la $\mathrm{I} 7^{\mathrm{e}}$ semaine pour I 2 enfants normaux, chiffre proche du risque de récurrence à terme de la trisomie $2 \mathrm{I}$ chez ces mères, évalué à IO-20 p. IoO dans les études rétrospectives (IO, II). La correction par rapport à la probabilité théorique résulte des avortements spontanés précoces. Nous n'avons étudié que 4 grossesses lorsque le père est porteur, dans ce cas le risque de récurrence à terme de la trisomie $2 \mathbf{I}$ a été évalué à moins de 5 p. Ioo [IO, II]. Le risque apparemment plus faible peut être expliqué par une évolution plus souvent létale des zygotes trisomiques 2 I entraînant l'élimination de la plupart des zygotes aneuplö̈des comme dans nos observations.

On doit aussi envisager les conséquences de ces anomalies sur la spermatogenèse entraînant une oligo-asthéno-tératospermie conduisant à une hypofertilité [I2].

Des stérilités ou une hypofertilité et peut-être l'évolution plus souvent létale des zygotes aneuploïdes lorsque le père porte l'anonalie permettent d'expliquer le faible nombre de translocations transmises par le père dans notre série. En effet, les couples sont venus consulter sachant qu'ils pouvaient transmettre une anomalie de structure, anomalie décelée soit à la suite de la naissance d'enfants malformés, soit à la suite d'avortements à répétition.

Dans d'autres cas l'anomalie avait été reconnue chez des collatéraux, mais 
les enquêtes familiales nous ont montré qu'il était plus difficile de faire une analyse chromosomique chez les hommes que chez les femmes diminuant donc ainsi le nombre des anomalies décelées chez des hommes.

Des études familiales plus poussées devraient permettre de mieux apprécier les différences qui peuvent apparaître sur la reproduction selon que l'anomalie est transmise par le père ou la mère.

L'histoire obstétricale des couples oì l'un des parents était porteur d'une inversion péricentrique nous a conduit à analyser le rôle de cette anomalie dans les échecs de la reproduction. Outre les couples venus pour un diagnostic prénatal nous avons pendant la même période réuni d'autres observations de couples avec les mêmes anomalies, examinés après avortements itératifs, ou naissances d'enfants malformés $\left[8, \mathrm{I}_{3}, \mathrm{I} 4\right]$.

Le premier point est que l'anomalie était portée par le père ro fois sur I4 ce qui contraste avec ce qui a été observé dans les translocations.

L.e tableau 4 montre 1'histoire obstétricale de ces couples.

TABLEAU 4

Histoire obstétricale

\begin{tabular}{|c|c|c|c|c|c|c|}
\hline Anomalie & Porteur & Stérilité & $\begin{array}{l}\text { Avortements } \\
\text { spontanés }\end{array}$ & $\begin{array}{l}\text { Ènfants } \\
\text { malformés }\end{array}$ & $\begin{array}{l}\text { Enfants } \\
\text { normaux }\end{array}$ & Diagnostic prénatal \\
\hline inv. 2 & $\begin{array}{l}\text { père } \\
\text { père } \\
\text { mère }\end{array}$ & & $\begin{array}{l}3 \\
1\end{array}$ & $\begin{array}{l}1 \\
1\end{array}$ & & $\begin{array}{l}46, \mathrm{XY} \\
46, \mathrm{XX} \text {, inv. } 2 \\
46, \mathrm{XY}\end{array}$ \\
\hline inv. 3 & père & & & 1 & & $\begin{array}{l}\text { aneusomie de } \\
\text { recombinaison }\end{array}$ \\
\hline inv. 5 & mère & & 1. & & & $46, \mathrm{XX}$, inv. 5 \\
\hline inv. 9 & $\begin{array}{l}\text { père } \\
\text { père } \\
\text { père } \\
\text { père } \\
\text { père } \\
\text { père } \\
\text { père } \\
\text { mère } \\
\text { mère } \\
\text { mère }\end{array}$ & $\begin{array}{l}19 \text { ans } \\
15 \text { ans } \\
7 \text { ans } \\
3 \text { ans }\end{array}$ & $\begin{array}{c}\text { 't } \\
\text { grossesse } \\
\text { extra-utérine } \\
1 \\
3(1 \text { Tri. } 8 \\
\text { inv. } 9)\end{array}$ & $\begin{array}{c}\text { Tri. } 21 \\
\text { Tri. } 21 \\
1 \\
1 \\
t(1-7), \text { inv. } 9\end{array}$ & $\begin{array}{l}1 \\
2 \\
3\end{array}$ & $\begin{array}{l}46, \mathrm{XY} \\
46, \mathrm{XX} \text {, inv. } 9 \\
\text { normal }\end{array}$ \\
\hline
\end{tabular}

Il apparaît que selon l'origine paternelle ou maternelle de l'inversion chromosomique les accidents de la reproduction sont différents. Alors que la fertilité féminine paraît peu modifiée, on remarque de longues périodes de stérilité du couple quand l'homme est porteur de l'inversion, en particulier dans l'inversion du 9. Il peut s'agir d'une perturbation directe de la spermatogenèse (spermogramme très perturbé). Des aneusomies de recombinaison par remaniement méiotique pourraient 
aussi être à l'origine de stérilité apparente par élimination précoce des zygotes avant l'implantation, mais nous n'en avons aucune preuve. Par contre, la fréquence des avortements cliniques reconnus, la découverte de trisomies associées à l'inversion dans ces avortements, la naissance d'enfants trisomiques 2 I chez ces couples et l'observation de translocations réciproques mettent en évidence l'importance d'un effet interchromosomique.

\section{CONCLUSIONS}

Le recrutement d'un laboratoire de diagnostic prénatal permet l'étude rétrospective et prospective de la reproduction de couples où l'un des géniteurs est porteur d'une anomalie de la structure chromosomique. Cette étude devrait permettre de mietrx cerner d'tune part les conséquences de ces anomalies sur les échecs de la reproduction et d'autre part d'essayer de comprendre pourquoi certaines anomalies (la trisomie 2I en particulier) conduisent soit à des arrêts précoces du développement, soit à une évolution jusqu'à terme.

L'analyse de ces premiers résultats montre que l'évolution au-delà de la $\mathbf{I}^{\mathrm{e}}$ semaine de gestation des zygotes déséquilibrés est rare et est le reflet de l'importance des arrêts précoces du développement décelables (avortements spontanés) ou non décelables.

La comparaison de l'histoire obstétricale permet de penser que les conséquences de ces anomalies peuvent être différentes en fonction du géniteur porteur de l'anomalie. Lorsque l'anomalie est portée par la mère la fécondité semble normale. Par contre, lorsque l'anomalie est portée par le père on constate des stérilités qui peuvent être expliquées soit par une action quantitative directe sur la spermatogenèse, soit par des fécondations répétées de zygotes déséquilibrés éliminés très tôt, ceci est surtout net dans les inversions péricentriques.

Il est possible en outre que dans le cas d'anomalies compatibles avec la venue à terme (trisomie 2I par exemple) l'évolution vers un avortement spontané soit plus fréquente lorsque la translocation est portée par le père.

Colloque D. G. R. S. T., Biologie de la Procréation, Paris, 7-8 mars 1975.

\section{REMERCIEMEN'TS}

Ce travail a bénéficié de l’aide de la D. G. R. S. T., contrat no 73-7-r659.

\section{SUMMARY}

PROSPECTIVE, STUDY OF HUMAN CHROMOSOMAL, FACTORS OF QUALITY OF GAME'TES

It scemed more casy to study chromosomal factors acting on the quality of gametes in carricrs of balanced chromosome structural anomaly. With genetic counselling for prenatal diagnosis it is possible to follow prospectively the outcome of pregnancies in these couples. 
The analysis of preliminary results shows that:

I. Most of the unbalanced zygotes stopped early their development and are eliminated before the 16 th week of pregnancy;

2. Among carriers referred for Robertsonian and reciprocal translocations one tenth only were male; this may reflect more severe consequences of structural anomalies leading to oligospermia and sterility, and early abortions; this is illustrated by the findings in $\mathbf{I} 4 q 2 \mathrm{I} q$ translocations and in pericentric inversions of chromosomes 2 and 9 .

\section{RÉFÉRENCES BIBLIOGRAPHIQUES}

[I] Boué A., Boué J. G., I973. Évaluation des erreurs chromosomiques au moment de la conception Biomédecine, 18, 372 .

[2] Boué A., Boué J. G., I975. Chromosomes abnormalities and abortion. In : Physiology and Genetics of Reproduction. E. M. Courinho et F. Fuchs, Eds., Plenum, New York., 317-339.

[3] Boú́ A., BouÉ J. G., 1974. Les accidents chromosomiques de la reproduction. In : Journées Parisiennes de Pédiatrie, I $\mathrm{x}-22$.

[4] Bouḱ A., Bové J. G., LAZAR P., I975. The epidemiology of human spontaneous abortions with chromosomal anomalies. In : Biology and Pathology of aging gametes. R. J. BLANdAu, Ed. Karger, 330-348.

[5] Cure S., Boué J. G., Boué A., I974. Growth characteristics of human embryonic cell lines with chromosomal anomalies. Biomedecine, 21, 233-236.

[6] Boué J. G., Boué A., I973. Anomalies chromosomiques dans les avortements spontanés. Colloque sur les Accidents chromosomiques de la Reproduction. I. N. S. E. R. M., Paris, 29-53.

[7] Bouḱ J. G., Bouê A., I973. Intérêt en diagnostic prénatal des nouvelles techniques d'identification chromosomique dans les translocations et une aneusomie de recombinaison. Nouvelle Presse Médicale, 2, 3097-3102.

[8] Bouḱ J. G., Hrrschhorn K., Lucas M., Gautier M., Moszer M., Bach Ch., i974. Aneusomies de recombinaison, conséquences d'une inversion péricentrique d'un chromosome " 3 " paternel. Ann. Pédiatrie, 21, 567-573.

[9] Boué J. G., Bouḱ A., Deluchat C., Thepot F., I975. Pronostic génétique des translocations I4-2r dans le cadre du diagnostic prénatal. Lyon Médical, 233, 207-210.

[ro] Hamerton J. L., I97o. Robertsonian translocation : evidence on segregation from family studies. In : Human population cytogenetics P. A. Jacobs, W. H. Price et P. LARv. Eds, University Press Edinburgh, 64-80.

[it] Lejeune J., Dutrillaux B., de Grouchy J., I97o. Reciprocal translocation in human populations, a preliminary analysis. In : Human population cytogenetics, P. A. J Acoss, W. H. Price et P. LARv., Eds, University Press Edinburgh, 82-87.

[12] Hulten M., Lindsten J., I97o. The behaviour of structural aberrations at male meiosis. In : Human population cytogenetics, P. A. JAcoBs, W. H. Price et P. LARv, Eds, University Press Edinburgh, 24-6r.

[13] Leonard C., Hazael-Massieux P., Bocouet L., Larget-Piet L., Boué J., i975. Inversion péricentrique Inv (2) (pII qI3) dans des familles non apparentées. Humangenetik, 28, I2I-128.

[14] Boué J. G., Tatllemite J. L., Hazael-Massieux P., Leonard C., Boué A., i975. Association of pericentric inversion of chromosome 9 and reproductive failure in ten unrelated families. Humangenetik, (sous presse). 